\title{
Bootstrapped Newtonian Cosmology and the Cosmological Constant Problem
}

\author{
Roberto Casadio ${ }^{1,2, *,+}$ (D) and Andrea Giusti ${ }^{3,+}$ (D) \\ 1 Dipartimento di Fisica e Astronomia "A. Righi", Università di Bologna, via Irnerio 46, I-40126 Bologna, Italy \\ 2 I.N.F.N., Sezione di Bologna, IS-FLAG, via B. Pichat 6/2, I-40127 Bologna, Italy \\ 3 Department of Physics and Astronomy, Bishop's University, 2600 College Street, \\ Sherbrooke, QC J1M 1Z7, Canada; agiusti@ubishops.ca \\ * Correspondence: casadio@bo.infn.it \\ + These authors contributed equally to this work.
}

check for updates

Citation: Casadio, R.; Giusti, A. Bootstrapped Newtonian Cosmology and the Cosmological Constant Problem. Symmetry 2021, 13, 358. https://doi.org/10.3390/sym13020358

Academic Editors: Kazuharu Bamba and $\mathrm{Yi}$-Fu Cai

Received: 14 January 2021

Accepted: 19 February 2021

Published: 22 February 2021

Publisher's Note: MDPI stays neutral with regard to jurisdictional claims in published maps and institutional affiliations.

Copyright: (C) 2021 by the authors. Licensee MDPI, Basel, Switzerland. This article is an open access article distributed under the terms and conditions of the Creative Commons Attribution (CC BY) license (https:// creativecommons.org/licenses/by/ $4.0 /)$.

\begin{abstract}
Bootstrapped Newtonian gravity was developed with the purpose of estimating the impact of quantum physics in the nonlinear regime of the gravitational interaction, akin to corpuscular models of black holes and inflation. In this work, we set the ground for extending the bootstrapped Newtonian picture to cosmological spaces. We further discuss how such models of quantum cosmology can lead to a natural solution to the cosmological constant problem.
\end{abstract}

Keywords: dark energy; quantum gravity; cosmological constant

\section{Introduction}

The standard model of the present Universe, often referred to as the $\Lambda$-Cold Dark Matter $(\Lambda C D M)$ model, allows us to include the current accelerated expansion of the Universe, confirmed by type I supernovae surveys [1], in the broader scheme of general relativity. However, this is carried out at the price of adding an ad hoc cosmological constant term $\Lambda$ in the Einstein field equations or by assuming the existence of an exotic fluid, dubbed dark energy, of which the pressure $p$ and energy density $\rho$ are such that $\rho+p \approx 0$. Throughout the work we set the speed of light $c=1$, while we express Newton's constant $G_{\mathrm{N}}=\ell_{\mathrm{p}} / m_{\mathrm{p}}$ and Planck's constant $\hbar=\ell_{\mathrm{p}} m_{\mathrm{p}}$ in terms of the Planck length $\ell_{\mathrm{p}}$ and the Planck mass $m_{\mathrm{p}}$. Further, we use $\simeq$ to denote that two quantities are equal up to order-one multiplicative factors.

Corpuscular gravity [2-4] is a quantum description based on the classicalization scheme [5] applied to the gravitational interaction. In some detail, the ultraviolet incompleteness of the Einstein-Hilbert gravity is conjectured to be cured by the formation of extended bound states of a large number of soft (off-shell) gravitons in very energetic processes (for interesting cases with a few gravitons involved see Reference [6]). These gravitons are prone to escape the bound state by quantum depletion, since they are very weakly coupled with each other and to other fields, but the large occupation endows the collective quantum state with a quasi-classical behaviour. According to this paradigm, a black hole of mass $M$ is understood as a marginally bound state of $N \simeq M^{2} / m_{\mathrm{p}}^{2}$ off-shell gravitons of typical momentum $\epsilon_{\mathrm{G}} \simeq m_{\mathrm{p}} / \sqrt{N}$ interacting with each other with an effective coupling $\alpha_{\mathrm{G}} \simeq 1 / N$. Since the collective coupling $N \alpha_{\mathrm{G}} \simeq 1$, the bound state cannot hold on to its constituents and will slowly lose components by the process of quantum depletion. The depletion of constituent gravitons represents the quantum precursor of the semiclassical Hawking evaporation as a consequence of the softness of the constituents and combinatorics. In fact, the master equation of the Hawking emission is recovered in the semiclassical limit of the theory, i.e., for $N \rightarrow \infty$ and $\ell_{\mathrm{p}} \rightarrow 0$, with the length scale

$$
L=\sqrt{N} \ell_{\mathrm{p}}
$$


and $\hbar$ kept fixed and finite. In the language of Bose-Einstein condensates, a "corpuscular" black hole is a condensate of gravitons on the verge of a quantum phase transition, which remains at the critical point throughout the evaporation process, up until the quantum break-time of the system. This "quantum criticality" provides nearly-gapless Bogoliubov modes (with energy gap $\Delta \epsilon \sim 1 / N$ ) accounting for the entropy of the black hole. A similar scenario can be derived from the Hamiltonian approach to gravity for the gravitational collapse of compact spherically symmetric distributions of matter [7]. Further, it is worth noting that this peculiar quantum description of black holes is strictly intertwined with the generalised uncertainty principle [8] and it has interesting implications for black-hole echoes [9].

Other space-times equipped with horizons have been studied within this scenario. In particular, one can apply arguments similar to the one above for black holes to the de Sitter space [3,10-14], embedding inflation and the late-time evolution of the Universe within a single theoretical paradigm. This corpuscular picture of the Universe further allows one to explain the dark matter phenomenology as the response of the cosmological condensate of gravitons, responsible for the quasi-de Sitter behaviour at a large scale, to the local presence of impurities, i.e., galaxies and (possibly) clusters of galaxies. Additionally, the corpuscular Hamiltonian approach [7] for cosmological spaces provides a quantum exclusion principle for the de Sitter space [3,10] and phantom energy [15].

In a nutshell, bootstrapped Newtonian gravity [16-20] is an approach aimed at providing a more detailed field-theoretic realisation for the quantum corpuscular theory of (spherically symmetric) compact sources. This approach has at its core the idea that, if quantum effects were negligible, starting from a field theory reproducing Newton's gravity, one should be able to recover general relativistic configurations by reconstructing (leading-order) non-linearities out of the coupling of each constituent graviton with the collective state. However, quantum effects are not assumed to be negligible and departures from general relativity are therefore expected (see, e.g., References $[4,16,17]$ for more details). This procedure represents, to some extent, an analogue of Deser's conjecture that the Einstein-Hilbert action in the vacuum should be obtained from a Fierz-Pauli Lagrangian for spin 2 fields in Minksowski space-time by adding suitable self-coupling terms [21,22]. Our approach instead focuses on the interplay between non-linearities and quantum physics in order to determine possible quantum corrections to the predictions of general relativity for compact but large self-gravitating systems in the strong field regime, for which the matter content is also far from the quantum vacuum.

In this work, we lay the basis for an extension of the bootstrapped Newtonian picture to cosmology, where the much simpler homogenous and isotropic spaces allow us to analyse also higher order terms. Furthermore, we show how this novel paradigm provides a natural solution to the cosmological constant problem.

\section{Corpuscular Models}

Let us start from the assumption that matter and the corpuscular state of gravitons together must reproduce the Einstein equations. In particular, we will focus on the Hamiltonian constraint corresponding to the time-reparameterisation invariance of the theory (the Hamiltonian constraint also plays the role of ensuring total energy conservation in a suitable sense)

$$
H_{\mathrm{M}}+H_{\mathrm{G}}=0,
$$

where $H_{\mathrm{M}}$ is the matter energy and $H_{\mathrm{G}}$ the analogue quantity for the graviton state. We recall that local gravity being attractive in general implies that $H_{\mathrm{G}} \leq 0$, although this is not true for the graviton self-interaction $[7,16]$.

\subsection{Corpuscular De Sitter and Black Holes}

The corpuscular models of gravity of References [2-4] were first introduced for describing black holes but are perhaps easier to obtain in cosmology, as the two systems share 
a fundamental "critical" condition. In order to have the de Sitter space-time in general relativity, one must assume the existence of a cosmological constant term, or vacuum energy density $\rho$, so that the Friedman equation (for spatially flat Universe) reads

$$
3\left(\frac{\dot{a}}{a}\right)^{2}=8 \pi G_{\mathrm{N}} \rho .
$$

Upon integrating on the volume inside the Hubble radius

$$
L=\frac{a}{\dot{a}},
$$

we obtain

$$
L \simeq G_{N} L^{3} \rho \simeq G_{N} M .
$$

This relation looks like the expression of the horizon radius for a black hole of ADM mass $M$ and is the reason it was conjectured that the de Sitter space-time could likewise be described as a condensate of gravitons [3].

One can describe the ideal corpuscular model as a condensate of $N$ (soft off-shell) gravitons of typical Compton length equal to $L$ in Equation (1). Then, since $M=N \epsilon_{\mathrm{G}}$ and $\epsilon_{\mathrm{G}}=\hbar / L$, one can infer that

$$
M=N \frac{\ell_{\mathrm{p}} m_{\mathrm{p}}}{L}=\sqrt{N} m_{\mathrm{p}},
$$

in agreement with Equation (5). This shows that for a macroscopic black hole, or de Sitter universe, one needs a huge number $N \gg 1$. Since the above corpuscular relations are more realistically expected to hold only approximately, we will assume that the energy $M$ is exactly given in terms of the fixed number of gravitons $N$ by Equation (6), whereas the Compton length of the gravitons denoted by $L$ henceforth can be (slightly) different from the Hubble value in Equation (1), which we will denote with $L_{N}=G_{\mathrm{N}} M=\sqrt{N} \ell_{\mathrm{p}}$. We thus write the Compton length as

$$
L=\xi^{-1} \sqrt{N} \ell_{\mathrm{p}} \equiv \xi^{-1} L_{N},
$$

where $\xi$ is a positive constant of order one which will play a significant role in the following analysis. In particular, we anticipate that we will find $L \gtrsim L_{N}$, that is $\xi \lesssim 1$, so that the gravitons must spread outside the Hubble radius $L_{N}$.

\section{2. "Post-Newtonian" Corpuscular Models}

Let us refine the above corpuscular description. In References $[7,16]$, it was shown that the maximal packing condition, which yields the scaling relations (7) for a black hole actually follow from the energy balance (2) when matter becomes totally negligible. If $H_{\mathrm{M}}=0$, one is left with

$$
H_{\mathrm{G}}=U_{\mathrm{N}}+U_{\mathrm{PN}}=0,
$$

where $H_{\mathrm{G}}$ is given by the sum of the negative (of course, we must assume the gravitational interaction is attractive and it is then important to keep track of minus signs in the following expressions) Newtonian energy [7]

$$
U_{\mathrm{N}} \simeq N \epsilon_{\mathrm{G}} \simeq-N \frac{\ell_{\mathrm{p}} m_{\mathrm{p}}}{L} \simeq-\frac{\ell_{\mathrm{p}} M^{2}}{L m_{\mathrm{p}}},
$$


and the positive "post-Newtonian" contribution [7]

$$
U_{\mathrm{PN}} \simeq N \frac{\sqrt{N} \ell_{\mathrm{p}}^{2} m_{\mathrm{p}}}{L^{2}} \simeq \frac{\ell_{\mathrm{p}}^{2} M^{3}}{L^{2} m_{\mathrm{p}}^{2}} .
$$

One therefore immediately recovers the scaling relation (7) from Equation (8), regardless of whether $L$ is the horizon radius of the de Sitter universe or of a black hole.

\section{Bootstrapped Newtonian Series}

Let us now note that

$$
U_{\mathrm{N}} \simeq\left(-\frac{\sqrt{N} \ell_{\mathrm{p}}}{L}\right) M=-\xi M,
$$

where $\xi$ was introduced in Equation (7), and

$$
U_{\mathrm{PN}} \simeq-\xi U_{\mathrm{N}} \simeq \frac{L_{N} M}{L^{2}},
$$

where we remark that $L$ is the Compton length of gravitons associated with either the horizon radius $L_{N}$ of the de Sitter universe or of a black hole. This leads us to conjecture that the complete form of the gravitational Hamiltonian to all orders in the $\xi$ expansion is given by

$$
H_{\mathrm{G}} \simeq M \sum_{n=1}^{\infty} g_{n}(-\xi)^{n},
$$

where we notice that $g_{1} \simeq g_{2} \simeq 1$ and the alternating signs above are precisely due to the attractiveness of gravity. This sort of expansion is strictly related to the argument of resurgence in QFT. In particular, the values of $g_{n}$ should arise directly from this argument (see, e.g., Reference [23]).

For a black hole, a matter contribution is needed and we cannot have $H_{\mathrm{G}}=0$. In particular, assuming the scaling relation (7), we note in passing that Equation (13) would reproduce the Newtonian expectation (we recall that the horizon radius can in fact be interpreted in terms of Newtonian gravity as the size of a source with escape velocity equal to the speed of light), that is

$$
H_{\mathrm{G}} \simeq U_{\mathrm{N}},
$$

for $g_{n} \simeq e \xi^{1-n} / n !(e-1)$ (Note that in this "Newtonian" case, the condition $g_{1} \simeq g_{2} \simeq 1$ does not hold.) Equation (2) then yields $H_{\mathrm{M}} \simeq U_{\mathrm{N}}$ with this expression of the coefficients $g_{n}$.

One more naive observation is that the typical momentum of the constituent gravitons scales as $1 / L$ which, in turn, is related to the spectral representation of the differential operator $\mathrm{d} / \mathrm{d} r$. Hence, one could view the exponential Equation (13) as the realisation of the black hole quantum state in infinite derivative gravity [24]. More precisely, one can see that the Schwarzschild metric (or quantum modifications thereof, and perhaps quantum general relativity as a whole), should be recovered as an infinite derivative theory in flat space-time (where the corpuscular picture is naturally formulated). Again, this is in line with bootstrapped Newtonian gravity [16,17] and Deser's works [21,22].

From now on we shall focus on pure gravity and cosmology, for which Equation (13) is more suitable. Let us then try to keep the analysis of Equation (13) as general as possible. We only require $g_{n}>0$ in the series (13) and the "on-shell" condition

$$
H_{\mathrm{G}}(L)=0 \quad \text { for } \quad L=\bar{L}=\xi^{-1} L_{N} ，
$$


with $\xi>0$ (and of order one) in the scaling relation (7). First of all, the series (13) converges provided the coefficients $g_{n}$ satisfy the inequality

$$
g_{n+1}<\xi^{-1} g_{n} .
$$

Note that, for our purpose, convergence is strictly required only for $L=\bar{L}$. Assuming however the series converges for $L$ at least in a neighbourhood of $\bar{L}$, the Hamiltonian $H_{\mathrm{G}}$ must be a smooth function of $L$ around $\bar{L}$, that is

$$
\left.\frac{\partial^{k} H_{\mathrm{G}}}{\partial L^{k}}\right|_{L=\bar{L}}=\left.M \sum_{n=1}^{\infty} g_{n} \frac{\partial^{k}}{\partial L^{k}}\left(-\frac{L_{N}}{L}\right)^{n}\right|_{L=\bar{L}},
$$

for some integers $1 \leq k \leq K$. Approaching the cosmological (or black hole) horizon, the contributions of nonlinear order of the theory will become of the same order of the Newtonian one, signalling the beginning of the full non-perturbative regime of gravity. This suggests that, not only $g_{1} \simeq g_{2} \simeq 1$ but also $g_{n}=\mathcal{O}(1)$ for all $n \geq 1$ (when $L$ approaches $L_{N}$ ), which is indeed consistent with Equation (8). Putting together this observation with the condition in Equation (16), the convergence of the above series requires $\xi$ to be smaller of an $\mathcal{O}(1)$ constant, which depends on the specific behaviour of the sequence of coefficients $g_{n}$. We remark that we always assume that the energy $M$ is fixed by the number of gravitons according to Equation (6) and it therefore does not depend on the Compton length $L$ (again for values around $L_{N}$ ). We then expect to obtain (slightly) more restrictive conditions for the coefficients $g_{n}$.

At this point, it is more interesting to consider other quantities of physical interest, and employ their relations with $H_{\mathrm{G}}$, in order to obtain further conditions on the coefficients $g_{n}$. For instance, we know that the pressure $p=-\rho$ in pure de Sitter space-time. From

$$
\begin{aligned}
p=\left.\frac{1}{4 \pi L^{2}} \frac{\partial H_{\mathrm{G}}}{\partial L}\right|_{L=\bar{L}} & =\left.\frac{M}{4 \pi \bar{L}^{2}} \sum_{n=1}^{\infty} g_{n} \frac{\partial}{\partial L}\left(-\frac{L_{N}}{L}\right)^{n}\right|_{L=\bar{L}} \\
& =-\frac{M}{4 \pi \bar{L}^{3}} \sum_{n=1}^{\infty} n g_{n}(-\xi)^{n},
\end{aligned}
$$

where we again used Equation (6). On considering the first two terms only $(n=1,2)$ and $H_{\mathrm{G}}=0$ up to the same order, that is $g_{1}=\xi g_{2}$, one finds

$$
p=-\frac{M g_{2} \xi^{2}}{4 \pi \bar{L}^{3}}=-\frac{g_{2} m_{\mathrm{p}} \xi^{5}}{4 \pi \ell_{\mathrm{p}}^{3} N} .
$$

From Equations (5), (6) and (15), we find

$$
\rho=\frac{M}{4 \pi \bar{L}^{3}}=\frac{m_{\mathrm{p}} \xi^{3}}{4 \pi \ell_{\mathrm{p}}^{3} N},
$$

so that $p=-\rho$ implies

$$
g_{2}=\xi^{-2}=g_{1}^{2} .
$$

Upon replacing this solution into Equation (13), we obtain the (slightly) off-shell Hamiltonian

$$
H_{\mathrm{G}}(L)=M\left[-\xi^{-1}\left(\frac{\sqrt{N} \ell_{\mathrm{p}}}{L}\right)+\xi^{-2}\left(\frac{\sqrt{N} \ell_{\mathrm{p}}}{L}\right)^{2}+\sum_{n=3}^{\infty} g_{n}\left(-\frac{\sqrt{N} \ell_{\mathrm{p}}}{L}\right)^{n}\right],
$$

where the higher-order terms should progressively capture all the non-linearities of the quantum theory of gravity. By looking at Equation (22), we can furthermore conjecture 
that $g_{n} \simeq \xi^{-n}$, but the precise values of such coefficients of interest to us will have to entail the specific features of the actual quantum state of the Universe. The precise determination of the $g_{n}$ for the observable Universe hence requires further investigation, as they will necessarily depend on the actual matter distribution and time evolution, which led to the present state.

In particular, since our Universe is not empty now, we can employ a more general equation of state for the purpose of improving our estimates of the coefficients $g_{n}$. In fact, we can easily generalise the above picture for any equation of state of the form

$$
p=-\omega \rho,
$$

where $0<\omega \leq 1$ is a constant. This yields

$$
\frac{g_{2}}{\omega}=\xi^{-2}=\frac{g_{1}^{2}}{\omega^{2}} .
$$

We can then conjecture that $g_{n} \simeq \omega \xi^{-n}$. Note that the pure dust case $p=0$ turns out to be rather peculiar in this scenario. Indeed, there are two possible configurations that allow Equation (18) to reduce to $p=0$ : (i) $g_{n}=0, \forall n \geq 2$, i.e., the purely Newtonian case, in agreement with the Newtonian derivation of the Friedmann equation (3); (ii) $\xi=0$, which implies $N \rightarrow 0$, thus reducing to Minkowski space.

We can finally notice that, upon employing the present value for the Hubble size of the Universe, that is $L_{N} \simeq \bar{L} \simeq 10^{27} \mathrm{~m}$, Equation (20) yields the correct order of magnitude for the present total energy density

$$
\rho=\frac{M}{4 \pi \bar{L}^{3}} \simeq 10^{-28} \frac{\mathrm{kg}}{\mathrm{m}^{3}},
$$

without any further assumptions. From Equation (20) and the definition (7) we can additionally estimate the observed value

$$
\xi=\left(4 \pi \rho \frac{\ell_{\mathrm{p}}}{m_{\mathrm{p}}} L_{N}^{2}\right)^{1 / 3} \simeq 0.9<1,
$$

which implies that the Compton length of the background gravitons $L>L_{N}$, as anticipated. Equation (24) can then be implemented to infer the equation of state parameter $\omega \simeq \xi g_{1} \simeq$ $0.9 g_{1}$ and $g_{2} \simeq g_{1} / \xi \simeq 1.1 g_{1}$, where we again recall that $g_{1} \simeq 1$. In fact, all of these final estimates are consistent with our previous assumption that the coefficients $g_{n}=O(1)$.

The above (somewhat preliminary) analysis shows that the apparently unnatural small value of the cosmological constant is indeed consistent with the quantum state of the observable Universe obtained from the bootstrapped Newtonian approach (see also References [3,25]). Moreover, a detailed comparison with cosmological measurements can be employed in order to determine the fine details of the quantum state of gravitons forming the background of the present quasi-de Sitter Universe. Of course, one would need to consider the time evolution of the Universe from the surface of the last scattering in order to determine the particle horizon. We leave such a question for further developments.

\section{Concluding Remarks}

Inspired by the corpuscular theory of gravity, in which the (matter empty) de Sitter Universe is described by a bound state of gravitons, we have shown how one can express the gravitational Hamiltonian $H_{\mathrm{G}}$ for a cosmological space to all "post-Newtonian" orders as the Newtonian energy of the leading order $U_{\mathrm{N}}$ multiplied by a power series in the quantity $\xi=L_{N} / L$, where $L$ is the Compton length of the bound gravitons and $L_{N}$ is the Hubble radius of the Universe determined by its (vacuum) energy $M$. In other words, similarly to Deser's classical argument [21,22], one can reconstruct the full quantum Hamiltonian of cosmology by supplying ("bootstrapping") the Newtonian potential energy 
with the energies due to particle self-interactions within the marginally bound state. At the fundamental level, this further suggests that general relativity might be recovered as an infinite derivative field theory on a flat space-time, although quantum corrections are naturally expected to introduce deviations from the classical configurations, which will result in different values of the coefficients in the "post-Newtonian" expansion (13).

A crucial role in our analysis is played by the parameter $\xi$, which measures the (slight) departure from the ideal corpuscular relation $L=L_{N}$. A functional behaviour for the coefficients of the bootstrapped Newtonian expansion of $H_{\mathrm{G}}$ in powers of $\xi^{-1}$ around the ideal de Sitter case with $\omega=1$ was then obtained in Equation (22). In order to accommodate for our non-empty observable Universe, we allowed for a more general equation of state. In particular, if one sets the radius $L_{N} \simeq L$ of the cosmological horizon to the present size of the Universe, the corpuscular Hamiltonian constraint of cosmology yields an energy density that matches the observed one associated with the cosmological constant provided the parameter $\xi \simeq 0.9<1$. This implies that $L \gtrsim L_{N}$ and the gravitons spread slightly beyond the measured Hubble size. Hence, this prediction for the vacuum energy density coming solely from the observed size of the Universe and the corpuscular quantum description of cosmological spaces can arguably be regarded as leading to a natural solution of the cosmological constant problem.

Author Contributions: All authors have contributed equally, read and agreed to the published version of the manuscript.

Funding: This research received no external funding.

Institutional Review Board Statement: Not applicable for studies not involving humans or animals.

Informed Consent Statement: Not applicable for studies not involving humans.

Data Availability Statement: Not applicable for studies not reporting any data.

Acknowledgments: R.C. is partially supported by the INFN grant FLAG. A.G. is supported by the Natural Sciences and Engineering Research Council of Canada (Grant No. 2016-03803 to V. Faraoni) and by Bishop's University. This work has been carried out in the framework of activities of the Italian National Group for Mathematical Physics (Gruppo Nazionale per la Fisica Matematica (GNFM), Istituto Nazionale di Alta Matematica (INdAM)) and COST action Cantata.

Conflicts of Interest: The authors declare no conflict of interest.

\section{References}

1. Perlmutter, S.; Aldering, G.; Goldhaber, G.; Knop, R.A.; Nugent, P.; Castro, P.G.; Deustua, S.; Fabbro, S.; Goobar, A.; Groom, D.E.; et al. Measurements of $\Omega$ and $\Lambda$ from 42 high redshift supernovae. Astrophys. J. 1999, 517, 565. [CrossRef]

2. Dvali, G.; Gomez, C. Black Hole's Quantum N-Portrait. Fortsch. Phys. 2013, 61, 742. [CrossRef]

3. Dvali, G.; Gomez, C. Quantum Compositeness of Gravity: Black Holes, AdS and Inflation. J. Cosmol. Astropart. Phys. 2014, 2014, 023. [CrossRef]

4. Giusti, A. On the corpuscular theory of gravity. Int. J. Geom. Meth. Mod. Phys. 2019, 16, 1930001. [CrossRef]

5. Dvali, G.; Giudice, G.F.; Gomez, C.; Kehagias, A. UV-Completion by Classicalization. J. High Energy Phys. 2011, 8, 108. [CrossRef]

6. Blas, D.; Camalich, J.M.; Oller, J.A. Scalar resonance in graviton-graviton scattering at high-energies: The graviball. arXiv 2020, arXiv:2009.07817.

7. Casadio, R.; Giugno, A.; Giusti, A. Matter and gravitons in the gravitational collapse. Phys. Lett. B 2016, 763, 337. [CrossRef]

8. Buoninfante, L.; Luciano, G.G.; Petruzziello, L. Generalized Uncertainty Principle and Corpuscular Gravity. Eur. Phys. J. C 2019, 79, 663. [CrossRef]

9. Buoninfante, L. Echoes from corpuscular black holes. J. Cosmol. Astropart. Phys. 2020, 12, 041. [CrossRef]

10. Casadio, R.; Giugno, A.; Giusti, A. Corpuscular slow-roll inflation. Phys. Rev. D 2018, 97, 024041. [CrossRef]

11. Cadoni, M.; Casadio, R.; Giusti, A.; Mück, W.; Tuveri, M. Effective Fluid Description of the Dark Universe. Phys. Lett. B 2018, 776, 242. [CrossRef]

12. Cadoni, M.; Casadio, R.; Giusti, A.; Tuveri, M. Emergence of a Dark Force in Corpuscular Gravity. Phys. Rev. D 2018, 97, 044047. [CrossRef]

13. Tuveri, M.; Cadoni, M. Galactic dynamics and long-range quantum gravity. Phys. Rev. D 2019, 100, 024029.

14. Cadoni, M.; Tuveri, M.; Sanna, A.P. Long-Range Quantum Gravity. Symmetry 2020, 12, 1396. [CrossRef] 
15. Casadio, R.; Giugno, A.; Giusti, A.; Faraoni, V. Is de Sitter space always excluded in semiclassical f(R) gravity? J. Cosmol. Astropart. Phys. 2019, 6, 5. [CrossRef]

16. Casadio, R.; Giugno, A.; Giusti, A.; Lenzi, M. Quantum corpuscular corrections to the Newtonian potential. Phys. Rev. D 2017, 96, 044010. [CrossRef]

17. Casadio, R.; Lenzi, M.; Micu, O. Bootstrapping Newtonian gravity. Phys. Rev. D 2018, 98, 104016. [CrossRef]

18. Casadio, R.; Lenzi, M.; Micu, O. Bootstrapped Newtonian stars and black holes. Eur. Phys. J. C 2019, 79, 894. [CrossRef]

19. Casadio, R.; Giusti, A. The role of collapsed matter in the decay of black holes. Phys. Lett. B 2019, 797, 134915. [CrossRef]

20. Casadio, R.; Kuntz, I. Bootstrapped Newtonian quantum gravity. Eur. Phys. J. C 2020, 80, 581. [CrossRef]

21. Deser, S. Selfinteraction and gauge invariance. Gen. Rel. Grav. 1970, 1, 9. [CrossRef]

22. Deser, S. Gravity from self-interaction redux. Gen. Rel. Grav. 2010, 42, 641. [CrossRef]

23. Dorigoni, D. An Introduction to Resurgence, Trans-Series and Alien Calculus. Ann. Phys. 2019, 409, 167914. [CrossRef]

24. Myung, Y.S.; Park, Y.J.; The origin of regular Newtonian potential in infinite derivative gravity. arXiv 2017, arXiv:1709.04587.

25. Dvali, G. S-Matrix and Anomaly of de Sitter. Symmetry 2020, 13, 3. [CrossRef] 